\title{
INTERAÇÃO MEDICAMENTOSA: UM AGRAVO À SAÚDE FRAGILIZADA
}

\author{
INTERACTION OF MEDICINES: AGGRAVATED LESION IN HEALTH \\ Kledson Lopes Barbosa ${ }^{\mathrm{a}^{*}}$, Karina Crislane da Silva de Medeiros ${ }^{\mathrm{b}^{* *}}$ \\ kledsonlopesb@gmail.com ${ }^{\mathrm{a}}$, karina_krislane@hotmail.com ${ }^{\mathrm{b}}$ \\ Universidade Federal de Alagoas*, Faculdade Cathedral/I-Brás**
}

Data do recebimento do artigo: $14 / 04 / 2018$

Data do aceite: $30 / 05 / 2018$

\begin{abstract}
RESUMO
Introdução: A facilidade na compra de medicamentos isentos de prescrição (MIP’s) e a falta de informação são as causas mais comuns de interação medicamentosa em indivíduos que convivem em múltipla terapia medicamentosa. Sabe-se que a interação fármaco-fármaco pode ocorrer quando o efeito de um medicamento é alterado pela presença da substância de outro medicamento, ou de algum alimento, o que pode causar diminuição da sua eficácia ou até mesmo a sua toxicidade. Nesse sentido, duas motivações surgiram para o desenvolvimento dessa pesquisa: o valor clínico dado a interação medicamentosa e a importância da assistência farmacêutica na prevenção desse agravo. Objetivo: refletir sobre os potenciais agravos à saúde individual e pública a medida que aumenta-se o uso de múltiplos medicamentos, e avaliar a frequência de publicações acerca do tema bem como o tipo de informação veiculada por esses. Método: Trata-se de uma revisão narrativa realizada com base em periódicos nacionais e internacionais abordando assuntos relacionados ao tema. As bases consultadas para esta produção foram Bireme, LILACS, SciELO, Pubmed e portal de periódicos CAPES. Resultados: A pesquisa bibliográfica revelou que a adoção do uso racional de fármacos está relacionado com o aconselhamento do profissional farmacêutico que promove o uso seguro dos medicamentos, bem como, o esclarecimento dos processos farmacoterapêuticos. Conclusões: Através do estudo reforça-se a importância do farmacêutico frente à prevenção e gerenciamento dos casos de interação medicamentosa, de modo a averiguar criteriosamente as prescrições médicas antes da dispensação a fim de reorientar os pacientes ao uso seguro dos medicamentos prescritos.
\end{abstract}

Palavras-chaves: Assistência farmacêutica; interações medicamentosas; prescrição médica

\begin{abstract}
Introduction: The ease of buying prescription-free medicines (MIP) and a lack of information are common causes of drug interaction in individuals who coexist in multiple drug therapy. It is known that drug-drug interaction occurs when the effect of a drug is altered by the presence of the substance of another drug, or of some food, what can cause a decrease in its efficiency or even its toxicity. In this sense, two motivations emerged for the development of this research: the clinical value given to drug interaction and the importance of pharmaceutical assistance in the prevention of this disease. Objective: to reflect on potential individual and public health problems as the use of multiple medications increases, and to assess the frequency of publications on the subject as well as the type of information conveyed by them. Method This is a narrative review based on national and international journals addressing issues related to the topic. The databases consulted for this production were Bireme, LILACS, SciELO, Pubmed and CAPES journal portal. Results: The literature review revealed that the adoption of rational use of drugs is related to the advice of the pharmacist who promotes the safe use of medications, as well as clarification of pharmacotherapeutic processes. Conclusions: The study reinforces the importance of the pharmacist to the prevention and management of cases of drug interaction, to carefully assess pre-dispensing medical prescriptions in order to reorient patients to the safe use of prescribed drugs.
\end{abstract}

Keywords: Pharmaceutical care; drug interactions; doctor's prescription 


\section{INTRODUÇÃO}

Os eventos adversos proporcionados pela interação medicamentosa se tornaram um grande problema de saúde pública devido aos elevados riscos a saúde dos pacientes, bem como o ônus econômico causado as unidades de saúde durante a assistência médica prestada a esses indivíduos quando internados. ${ }^{1}$

Apesar da interação medicamentosa ser alvo de pesquisa, ainda falta muito a ser elucidado sobre seu mecanismo, bem como, se faz necessário o entendimento das características dos pacientes expostos a interação fármaco-fármaco. As principais causas para o surgimento dessa complicação são o aumento da idade, número de medicamentos prescritos e principalmente o desconhecimento dessa abordagem através do profissional médico. ${ }^{2,3}$

Sabe-se que a interação medicamentosa ocorre quando o efeito de um medicamento é alterado pela presença da substância de outro medicamento, ou de algum alimento, o que pode causar diminuição da sua eficiência ou até mesmo provocar sua toxicidade. As interações medicamentosas podem ser classificadas sob duas formas, a primeira como farmacocinéticas, que são as que causam modificação nos parâmetros de absorção, distribuição, metabolismo e excreção, a segunda, farmacodinâmicas que resulta em alterações dos sítios receptores: pré e pós-receptor, ou interações agonistas e antagonistas, respectivamente. ${ }^{4}$

Quanto às complicações em decorrência da interação medicamentosa, podem ser citadas as de menor gravidade, onde surgem efeitos clínicos restritos que não carecem de mudança na terapia medicamentosa, e a moderada, quando surge agravamento no quadro clínico sendo necessário mudar a terapia medicamentosa. E por fim, maior e/ou grave, que é quando a interação evolui para forma crônica, neste caso, é indispensável à intervenção médica para minorar as reações adversas graves. ${ }^{4,5}$

A facilidade na compra de medicamentos isentos de prescrição (MIP's) e a falta de informação são as causas mais comuns de interação medicamentosa em indivíduos que convivem em múltipla terapia medicamentosa, haja vista a per- da da atividade farmacológica do medicamento. ${ }^{6}$ Sendo assim, o conhecimento sobre interação medicamentosa é um artifício importante para evitar interações entre fármaco-fármaco e promover o tratamento seguro do paciente aumentanto sua qualidade de vida. ${ }^{7}$

Nesse sentido, duas motivações surgiram para o desenvolvimento dessa pesquisa: o valor clínico dado às interações medicamentosas e a importância da assistência farmacêutica na prevenção desse agravo. Diante disto, o objetivo do presente estudo foi refletir sobre os potenciais agravos à saúde individual e pública com o aumento do risco da incidência de interações medicamentosas a medida que aumenta-se o uso de múltiplos medicamentos, e avaliar a frequência de publicações acerca do tema bem como o tipo de informação veiculada por esses.

\section{METODOLOGIA}

O estudo trata-se de uma revisão narrativa de cunho qualitativa realizada com base em periódicos nacionais e internacionais abordando assuntos relacionados ao tema. As bases consultadas para esta produção foram Bireme, LILACS, SciELO, Pubmed e portal de periódicos CAPES. As buscas nas bases de dados nacionais foram realizadas utilizando os unitermos "assistência farmacêutica" "interação medicamentosa" ou "interação fármaco-fármaco" ou "uso multíplo de fármacos" e "prescrição médica”. Para as buscas internacionais: "pharmaceutical care" "drug interaction" "drug-drug interaction" "multidrug use" and medical prescription"

Para pesquisa bibliográfica, não houve restrições de data e idioma de publicação objetivando compilar estudos sobre interações medicamentosas e a importância da assistência farmacêutica. Foram selecionados para este estudo, o total 41 artigos publicados em diferentes bancos de dados. Os principais temas abordados podem ser visualizados na Tabela 1, cuja escolha foi respaldada, principalmente, sobre como essa pesquisa contribuiria para o avanço da assitência farmacêutica com destaque para a interação medicamentosa. 
Tabela 1: Resultados da pesquisa bibliográfica incluídas neste estudo

Tema das publicações

Assistência Farmacêutica

Boas Práticas Farmacêuticas

Interações Medicamentosas

Interações farmacológicas

Uso Racional de Medicamentos

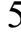

5

19

9

3

Fonte: Autor, 2017

À exceção das leis e portarias, as publicações utilizadas neste estudo corresponderam ao período de 1993 a 2017. Além disso, apesar de não haver restrições de idioma, observou-se que aleatoriamente foram selecionadas publicações nos idiomas português e inglês, o que revela ser um aspecto importante na escolha da literatura a ser citada, bem como, um aspecto crucial que torna as publicações facilmente encontráveis.

\section{ASSISTÊNCIA FARMACÊUTICA}

Foi através da constituição federal de 1988 que o direito a assistência em saúde, caracterizado como um direito fundamental de todos os cidadãos passou a ser um dever do estado. ${ }^{8}$ Logo depois, respaldada na Lei Orgânica de Saúde (LOS), a assistência farmacêutica tornou-se primordial para o esclarecimento do acesso aos medicamentos. ${ }^{9}$

O Brasil tem buscado por um serviço de excelência na assistência farmacêutica, e para isso, tem se apoiado no programa Farmácia Popular do Brasil, Política Nacional de Plantas Medicinais e Fitoterápicos e no Programa Nacional de Qualificação da Assistência Farmacêutica no SUS (QUALIFAR-SUS). É possível evidenciar que essas iniciativas fizeram e fazem até hoje parte do desenvolvimento do país, dada à questão da assistência farmacêutica, além do que, estes precursores, aliados ao investimento do governo tornaram possível ampliar o investimento das políticas farmacêuticas no Brasil. ${ }^{10}$

Gadelha e colaboradores ${ }^{10}$, enfatizam o quão se tornou imprescindível o uso racional de medicamentos por parte da população, haja vista que esses investimentos foram cruciais para a acessibilidade destes insumos ao longo dos anos. Sendo assim, a promoção do uso racional de medicamentos é uma estratégia que deve ser prioritariamente desenvolvida institucionalmente tendo à frente o farmacêutico, uma vez que, sua orientação é crucial para a prevenção das interações medicamentosas na dispensação.

Em um estudo bastante inspirador de Bruns e colaboradores ${ }^{11}$, os autores tornaram evidente que a assistência farmacêutica é primordial e essencial na melhoria da saúde e qualidade de vida da população, assim como, para o uso racional de medicamento e diminuição dos gastos em saúde pública. Essa questão justifica-se com maior relevância quando a Organização Mundial de Saúde ${ }^{12}$, retrata que $50 \%$ de todos os medicamentos prescritos, dispensados e vendidos são administrados inadequadamente. Nesse contexto, é urgente que o farmacêutico esteja desenvolvendo atividades de controle na farmácia, na dispensação e na orientação, que esteja inserido nas equipes de saúde a fim de melhorar os serviços de saúde disponíveis a população. ${ }^{11}$

\section{INTERAÇÃO MEDICAMENTOSA}

Interação medicamentosa é a resposta causada pelo uso simultâneo de diferentes fármacos, ou deste com alimento, bebida, agente químico ou ambiental. A maior preocupação para interação medicamentosa é quando surge um quadro exacerbado de toxicidade ou redução do efeito do fármaco, e como consequência, aparição de complicações adversas por não tratar a patologia do paciente em questão ${ }^{13,14}$, assim como, pode resultar em hospitalização e abandono do tratamento ${ }^{15}$.

Abordagens sobre interação medicamentosa tem ganhado destaque na literatura e causado bastante preocupação devido à utilização da polifarmácia, que se acredita estar ligada as causas de problemas relacionados a medicamentos PRM. ${ }^{13,16}$ Considerando as populações com maior prevalência de interações medicamentosas, destacam-se os idosos devido a maior predisposição 
destes indivíduos para o desenvolvimento de doenças crônicas, bem como, as sequelas adquiridas com o avanço da idade ${ }^{17,18}$, sendo assim, este grupo chega a consumir três vezes mais medicação que os jovens. ${ }^{19}$

Segundo Correr e colaboradores (20), as interações medicamentosas merecem atenção uma vez que elas podem ser confundidas com outras doenças, visto que incialmente surgem sintomas inespecíficos, devido a isto, comprometem mais ainda o estado de saúde do paciente. Cabe ressaltar, que os riscos para o surgimento da interação medicamentosa estão sujeitos e são diretamente proporcionais ao número de fármacos prescritos e utilizados pelo paciente, ou seja, quanto maior for o número de fármacos prescritos e, por conseguinte, inclusos no tratamento, maior serão as chances de interação medicamentosa devido esta politerapia. ${ }^{13}$ Victório e colaboradores ${ }^{21}$ propõem regular esse agravo através de estudo dos fármacos com maior prevalência nos casos de interação, como uma perspectiva para estimular uma política de atenção farmacêutica frente ações de farmacovigilância.

Análise das reações adversas entre fármacos descritas na literatura reportam que as interações medicamentosas representam $5 \%$ de todos os erros de medicação em polimedicados. Ademais, em muitas terapias, essas interações são previsíveis podendo ser evitadas e outras imprevisíveis. ${ }^{22,23}$ Nesse cenário preocupante, surge então a necessidade de implantar políticas públicas em saúde voltadas para o gerenciamento dos riscos eminentes ocasionados pelo múltiplo uso de medicamentos, visto que através das interações medicamentosas destacam-se a redução de ação do fármaco como também aumento da toxicidade. ${ }^{24}$

Dados da literatura indicam a interação medicamentosa como um fator nocivo de grande magnitude, visto que pode causar evolução negativa da condição clínica do paciente. Avalia-se também, que o alto risco de interação medicamentosa esteja presente em anemia aplástica, asma, arritmia cardíaca, diabetes, epilepsia, doenças altoimunes, doenças cardiovasculares, infecções, desordens psiquiátricas entre outras. ${ }^{25}$

Dois grupos são considerados susceptíveis ao desenvolvimento de interações medicamentosas, são eles: os hipertensos e diabéticos, haja vista a prevalência dessas doenças no Brasil. Dessa maneira, o tratamento dessas patologias envolve adoção de terapias consideradas complexas através de muitos fármacos razoavelmente novos no mercado farmacêutico, o que maximiza as chances de interação medicamentos, dada a questão de que as interações medicamentosas ainda não são totalmente esclarecidas. ${ }^{26}$ Nesse contexto, torna-se urgente o monitoramento das prescrições medidas, sejam elas de unidade de terapia intensa, farmácias ou drogarias, visto a grande quantidade de prescrições dispensadas nas UTI's bem como, a elevada rotatividade nos estabelecimentos comerciais de fármacos. ${ }^{4}$

\section{EVIDÊNCIAS DE INTERAÇÃO MEDICAMENTOSA}

Interação medicamentosa não se trata de um tema recente, e através da Figura 1 pode-se perceber que muitos estudos estão sendo desenvolvidos a cerca deste assunto, e, como resultado, a comunidade científica dispõe de inúmeras informações sobre interações medicamentosas, assim como, os potenciais medicamentos envolvidos nesse mecanismo.

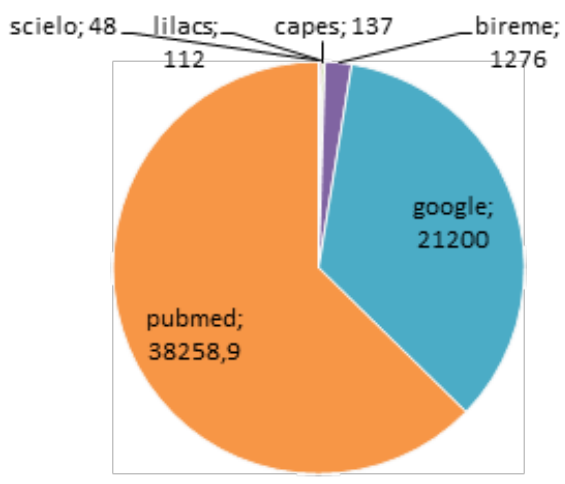

Figura 1: Número de publicações sobre interação medicamentosa em diferentes bibliotecas de circulação nacional e internacional

Fonte: Autor, 2017

Em relação à pesquisa bibliográfica, considera-se que as interações medicamentosas podem resultar em muitos agravos a saúde na população carente de informação, de assistência e mesmo aqueles assistidos dentro de uma unidade de saúde em constante monitoramento por profissionais da saúde. Via a esses fatores, enfatiza-se a necessidade da avaliação criteriosa sobre a administração múltipla de medicamentos. 
Os resultados obtidos nos bancos de dados (Figura 1) refletem o número de artigos encontrados em relação às palavras-chaves utilizadas nas buscas. Dessa forma, o número de artigos encontrados correspondentes as bases foram 48 SciELO, 112 LILACS, 137 Periódicos CAPES, 1.276 Bireme, 21.200 Google Acadêmico e 38.258,9 PubMed, totalizando $61.031,9$ artigos publicados em bacos de dados indexados de circulação nacional e internacional.

No banco de dados PubMed, o quantitativo de publicações foi bastante expressivo quando comparado aos demais, esse resultado pode ser justificado pelo fato de que essa plataforma se tornou a principal ferramenta de busca na área médica, $o$ que reflete em números a indexação de vários periódicos. Em segundo lugar, destaca-se a plataforma Google Acadêmico, o que pode ser diagnosticado como uma boa alternativa de busca bibliográfica, por se tratar de uma ferramenta de fácil acesso, praticidade e possuir bastante popularidade.

A Biblioteca Virtual em Saúde (Bireme) ocupou o terceiro lugar com 1.276 artigos publicados sobre o tema em questão, enquanto que nas demais bibliotecas virtuais foi observado que o SciE-
LO é a plataforma com menor número de publicações sobre o tema de interação medicamentosa. No caso do periódico CAPES, específico do Brasil, pode-se dizer que o mesmo se encontra em fase de popularidade, uma vez que foi estimulado a pouco tempo pelas universidades o seu uso para buscas bibliográficas. Já o LILACS reportou 112 artigos indexados. Independente de números, está claro que através dessas ferramentas virtuais é possível ter acesso a um vasto quantitativo de estudos que aumentam as possibilidades de conhecimento do campo de atuação do profissional farmacêutico, e que o conhecimento aqui explícito, foi o responsável por nortear a pesquisa e seu desenvolvimento.

\section{EFEITOS CAUSADOS POR INTERAÇÃO MEDICAMENTOSA}

Em um estudo sobre potenciais interações entre medicamentos, os autores indicaram vários fármacos como possíveis causadores de interação medicamentosa. Na tabela 2 estão apresentados os dados obtidos e publicados por Bandeira e Olivei$\mathrm{ra}^{27} \mathrm{em}$ seu estudo sobre interação medicamentosa usados na síndrome metabólica.

Tabela 2: Potenciais interações medicamentosas diagnosticadas na Relação Nacional de Medicamentos Essenciais (RENAME), 2014.

Fármacos

Hidroclorotiazida

Furosemida

Espironolactona

Atenolol, Carvedilol, Metropolol, Propanolol

Hidralazina

Verapamil
Fármacos

-Atenolol, Carvedilol, Metoprolol, Propranolol, Glibenclamida, Gliclazida, Metformina e Fluoxetina

-Hidralazina

-Atenolol, Carvedilol, metoprolol, Propranolol, Glibenclamida, Gliclazida, Metformina e Fluoxetina

-Atenolol, Carvedilol, metoprolol, Propranolol, Metformina e Fluoxetina

- Captopril, Enalapril e Losartana

-Anlodipino, Glibenclamida, Gliclazida, Metformina e

Fluoxetina

-Verapamil

-Sinvastatina

-Glibenclamida

-Fluoxetina

-Sinvastatina
Classificação da

IM

Moderada

Menor

Moderada

Moderada

Maior

Moderada

Maior

Moderada

Menor

Moderada

Maior 


\begin{tabular}{|c|c|c|}
\hline Anlodipino & $\begin{array}{l}\text {-Fluoxetina } \\
\text {-Sinvastatina }\end{array}$ & $\begin{array}{l}\text { Moderada } \\
\text { Maior }\end{array}$ \\
\hline \multirow[t]{2}{*}{ Captopril e Enalapril } & $\begin{array}{c}\text {-Losartana, Glibenclamida, Gliclazida, Metformina } \\
\text {-Losartana }\end{array}$ & Moderada \\
\hline & & Maior \\
\hline $\begin{array}{l}\text { Glibemclamida e } \\
\text { Gliclazida }\end{array}$ & -Fenofibrato, Sinvastatina, Fluoxetina & Moderada \\
\hline
\end{tabular}

Fenofibrato

-Sinvastatina

Maior

Fonte: Modificado de Bandeira e Oliveira (27).

Conforme mostra a Tabela 2, as interações medicamentosas referem-se ao uso concomitante de fármacos utilizados para síndrome metabólica, tais como hipertensão, diabetes e dislipidemia. Dentre os medicamentos apresentados, o atenolol, carvedilol, metoprolol e propanolol são indicados pelos autores como os anti-hipertensivos que mais promovem interação medicamentosa quando utilizados com os hipoglicemiantes, tais como, glibenclamida e metformina, promovendo efeito contrário ao tratamento, o que pode causar hipoglicemia ou hiperglicemia.

Recomendações do Mistério da Saúde ${ }^{17}$, prever que o uso de fluoxetina pode potencializar o efeito dos betabloqueadores, aumentando as chances de parada cardíaca. Sendo assim, ao utilizar dois fármacos onde um potencialize o efeito do outro, não pode ser considerado como efeito positivo, uma vez que potencializar o efeito do betabloqueador é o mesmo que trazer riscos à saúde do paciente. ${ }^{27}$

A administração de sinvastatina com fenofibrato ocasiona maior interação medicamentosa, e como consequência, podem surgir miosite, miopatia, dor e fraqueza ${ }^{17}$, além disso, sua interação medicamentosa ocasionada por verapamil e anlodipino aumenta sua toxicidade, causando maios problemas de saúde ao indivíduo em tratamento. ${ }^{17,27}$

Nesse contexto, torna-se estritamente necessário que a interação medicamentosa seja considerada como um importante indicador de saúde aos indivíduos em constante terapia múltipla. Sendo assim, conhecer a interação medicamentosa é o primeiro passo para o seu controle e prevenção no sentido de evitar riscos desnecessários, visando um tratamento seguro e tranquilo ao indivíduo.

\section{O FARMACÊUTICO E SUA CONTRIBUIÇÃO PARA O USO RACIONAL DE MEDICAMENTOS}

Com o passar dos anos, tornou-se indiscutível a atuação do farmacêutico nos serviços de atenção à saúde primária ${ }^{28}$, bem como, sua intervenção nas unidades de saúde, farmácias e drogarias. Quanto a assistência farmacêutica, deve-se considerar como responsabilidade do farmacêutico: avaliar a prescrição e averiguar os potenciais medicamentos para a interação medicamentosa. ${ }^{28,29}$

A partir da implementação de políticas públicas de saúde e da ascensão de acesso aos medicamentos essências, criado através da Política Nacional de Medicamentos (PNM) em 1998, tornou-se emergente a presença do farmacêutico nesses programas a fim de contribuir com orientação aos pacientes sob as prescrições médicas, visto o expressivo aumento na oferta de medicamentos no país. ${ }^{30,31}$

Todavia, em um estudo realizado pelo Ministério da Saúde, detectou-se que mesmo diante dos programas de distribuição de medicamentos gratuitos através do Sistema Único de Saúde (SUS), o farmacêutico concentra suas atividades em caráter logístico. Desenvolvendo mais atividades de gestão do que de farmacovigilância, por essa razão, acredita-se que a escassez de farmacêuticos no SUS, traz como consequência prejuízos a assistência médica, visto que é importante a dispensação de medicamentos em unidades básicas de saúde assistidas por esses profissionais. ${ }^{29}$

Entende-se que o farmacêutico deve desempenhar em unidades de atenção básica o cuidado com o paciente, auxiliando no tratamento de doenças sejam elas na fase aguda ou crônica, e, na prevenção de agravos através da orientação sobre as prescrições, bem como, estimular a edu- 
cação em saúde para que os indivíduos se tornem conscientes e esclarecidos sobre o uso múltiplo de medicamentos e sua relação com as reações adversas que possam surgir pelo uso inadequado dos medicamentos. ${ }^{29}$

No que diz respeito ao uso racional de fármacos, o farmacêutico é essencial para estimular o uso seguro dos medicamentos, visto que é um profissional que possui formação e governabilidade para esclarecimento dos processos farmacoterapêuticos, e, por conseguinte, o mais apto a desenvolver a educação em saúde sobre essa temática, cujo foco estar a margem do cuidado farmacêutico. ${ }^{32,33}$

Sabe-se que hoje em dia, todas as farmácias e drogarias devem funcionar com a presença de um farmacêutico responsável durante todo o seu expediente $^{34,35}$, cujas diretrizes são pautadas em órgãos de padrões internacionais tais como $\mathrm{Fe}$ deração Internacional de Farmácia ${ }^{36}$, Associação Nacional de Autoridades Reguladoras de Farmácias $^{37}$ e da Sociedade Farmacêutica da Austrália ${ }^{38}$, que tem como objetivo resguardar as atividades do farmacêutico para estimular a saúde, o autocuidado, uso racional de medicamentos e principalmente o sucesso farmacoterápico individual de cada paciente. ${ }^{35}$ Aliada a esta condição, espera-se que ao aproximar o farmacêutico dos pacientes, obtenha-se uma nova postura nas unidades de saúde e nos estabelecimentos comerciais, pois, o que normalmente se identifica é o desenvolvimento de atividades burocráticas, que afastam o farmacêutico do trabalho focado no paciente. ${ }^{39}$

No século passado, as atividades que o farmacêutico desempenhava estavam entre a disponibilização, preparação e validação de fármacos para o tratamento de diversas patologias conforme sua indicação. Logo então, eram responsáveis pela inspeção do que se produzia e do que comercializava, com o advento da industrialização e as tecnologias desenvolvidas, esse atributo teve expressiva diminuição, uma vez que várias formulas farmacêuticas se tornaram produzidas em escala industrial. ${ }^{40}$

Nas unidades de saúde onde profissionais médicos e enfermeiros são insuficientes, o farmacêutico pode ser uma alternativa para monitoramento na terapia de doentes crônicos. Dessa forma, enfatizam que o farmacêutico ocupa lugar de prestígio dentro do sistema de saúde devido sua transita- bilidade entre a população. Ademais, através de sua formação, é possível prevenir doenças através de recomendações no monitoramento de terapia. Dito isto, é necessário que a relação entre paciente e farmacêutico priorizem os problemas relacionados ao uso múltiplo de fármacos bem como, a prevenção das interações medicamentosas. ${ }^{41}$

\section{CONCLUSÕES}

Diante de tudo que foi dito, reforça-se a importância do farmacêutico frente à prevenção e gerenciamento dos casos de interação medicamentosa, de modo a averiguar criteriosamente as prescrições médicas antes da dispensação a fim de reorientar os pacientes ao uso seguro dos medicamentos prescritos. Nesse sentido, é claro o fato de que o impacto clínico farmacêutico através de sua intervenção trará qualidade no uso racional dos medicamentos quando o farmacêutico desenvolver atividades que tenham como foco a atenção primária a saúde, visto que o mesmo se divide em funções de assistência e administrativas.

Portanto, esta revisão narrativa demonstrou os riscos associado às combinações múltiplas de fármacos sem orientação adequada do farmacêutico, principalmente entre aqueles disponíveis pelo RENAME. Além disto, o presente estudo revelou ainda que há um grande número de publicações sobre interação medicamentosa, e que mesmo diante desse cenário, é algo muito negligenciado em todo o mundo, demonstrando dessa forma, o quão é indispensável o desenvolvimento de políticas públicas voltadas à redução desse risco.

\section{REFERÊNCIAS}

1. Moura C, Acurcio F, Belo N. Drug-drug interactions associated with length of stay and cost of hospitalization. Journal of Pharmacy and Pharmaceutical Sciences. 2009;12(3):266-272. DOI:10.18433/J35C7Z.

2. Gagne JJ, Maio V, Rabinowitz C. Prevalence and predictors of potential drug-drug interactions in Regione Emilia-Romagna, Italy. Journal of Clinical Pharmacy and Therapeutics. 2008;33(2):141-151. DOI:10.1111/j. 1365-2710.2007.00891.x.

3. Egger SS, Bravo AER, Hess L, Schlienger RG, Krähenbühl S. Age-related differences in the prevalence of po- 
tential drug-drug interactions in ambulatory dyslipidaemic patients treated with statins. Drugs and Aging. 2007;24(5):429-440. DOI:10.2165/00002512-20072405000006 .

4. Yunes LP, Coelho T de A, Almeida SM de. Principais Interações Medicamentosas Em Pacientes Da Uti-Adulto De Um Hospital Privado De Minas Gerais. R Bras Fram Hosp Serv. 2011;2(61):23-6.

5. Miyasaka LS, Atallah AN. Risk of drug interaction: combination of antidepressants and other drugs. Rev Saude Publica [Internet]. 2003;37(2):212-215. DOI: 10.1590/ s0034-89102003000200008.

6. Hooda R. Research and Reviews : Journal of Pharmacognosy and Phytochemistry Herbal Drug Interactions - A Major Safety Concern. 2016;4(1):54-8.

7. Kafeel H, Rukh R, Qamar H, Bawany J, Jamshed M, Sheikh $\mathrm{R}$, et al. Possibility of Drug-Drug Interaction in Prescription Dispensed by Community and Hospital Pharmacy. Pharmacology and Pharmacy. 2014;5(April):401-7. DOI: 10.4236/pp.2014.54048.

8. Brasil. Constituição (1988). Constituição da República Federativa do Brasil. Brasília: Senado Federal; 1988. Disponível em: www.planalto.gov.br/ccivil_03/constituicao/ constitui\%C3\%A7ao.htm. Acessado em 08 de Fevereiro de 2017a.

9. Brasil. Lei 8 080/1990. Disponível em: www.planalto.gov. br/ccivil_03/Leis/L8080.htm. Acessado em 08 de março de $2017 b$.

10. Gadelha CAG, Costa KS, Nascimento Júnior JM do, Soeiro OM, Mengue SS, Motta ML da, et al. PNAUM: integrated approach to Pharmaceutical Services, Science, Technology and Innovation. Revista de Saúde Pública [Internet]. 2016;50(supl 2):1-8. DOI:10.1590/s15188787.2016050006153.

11. Bruns S de F, Oliveira EA de, Oliveira MA, Luiza VL. O modelo de ambiguidade-conflito como ferramenta de análise dos desafios da Assistência Farmacêutica em João Pessoa (PB) TT - The ambiguity-conflict model as tool for the analysis of Pharmaceutical Services challenges in João Pessoa, Paraíba, Brazil. Saúde em Debate [Internet]. 2015;39(spe):64-75. DOI: 10.5935/01031104.2015S005311.

12. World Health Organization (WHO). Promoting rational use of medicines: core components. (WHO Policy Perspectives on Medicines No. 5). Geneva, 2002. Disponível em: <http://apps.who.int/iris/bitstream/10665/67438/1/ WHO_EDM_2002.3.pdf $>$. Acesso em: 10.11.2017.

13. Da Silva NMO, Carvalho RP, Bernardes ACA, Moriel P, Mazzola PG, Franchini CC. Avaliação de potenciais interações medicamentosas em prescrições de pacientes internadas, em hospital público universitário especializado em saúde da mulher, em Campinas-SP. Revista de Ciencias Farmaceuticas Basica e Aplicada. 2010;31(2):171-6.
14. Brasil. Ministério da Saúde. Secretaria de Tecnologia e Insumos Estratégicos. Departamento de Assistência Farmacêutica e Insumos Estratégicos. Formulário terapêutico nacional. Brasília: Ministério da Saúde; 2008a.

15. Dormann H, Sonst A, Müller F, Vogler R, Patapovas A, Pfistermeister B, et al. Adverse drug events in older patients admitted as an emergency: the role of potentially inappropriate medication in elderly people (PRISCUS). Deutsches Ärzteblatt international. 2013;110(13):2139DOI: 10.3238/arztebl.2013.0213.

16. Maria I, Sette F, Abrantes J, Barbosa A. Estudo de utilização de medicamentos na clínica médica. Rev Bras Farm. 2008;89(3):267-71.

17. Brasil. Ministério da Saúde. Secretaria de Ciência, Tecnologia e Insumos Estratégicos, Departamento de Assistência Farmacêutica e Insumos Estratégicos. Formulário Terapêutico 2010. Rename 2010. Brasília (DF): MS; 2010.

18. Melgaçao TB, Carrera JDS, Nascimento DEB Do, Maia CDSF. Polypharmacy and ocurrences of potential drug interactions. Rev para med [Internet]. 2010;25(1). DOI: org/10.1590/S0034-71672010000100023.

19. Antunes J de FS, Okuno MFP, Lopes MCBT, Campanharo CRV, Batista REA. Interação medicamentosa em idosos internados no serviço de emergência de um hospital universitário TT Drug interaction in elderly inpatients in the emergency department of a university hospital TT - Interacción medicamentosa en adultos mayores ingresa. REME rev min enferm [Internet]. 2015;19(4):907-12.DOI:10.5935/1415-2762.20150070.

20. Correr CJ, Pontarolo R, Ferreira LC, Baptistão SAM. Riscos de problemas relacionados com medicamentos em pacientes de uma instituição geriátrica. Revista Brasileira de Ciencias Farmaceuticas/Brazilian Journal of Pharmaceutical Sciences [Internet]. 2007;43(1):55-62, DOI:10.1590/S1516-93322007000100007

21. Victorio CJM, Castilho SR de, Nunes PHC. Identificação de potenciais problemas relacionados com medicamentos a partir da análise de prescrições de pacientes hipertensos. RevBras Farm. 2008;89(3):233-5.

22. Cedraz KN, Junior MCS. Identificação e caracterização de interações medicamentosas em prescrições médicas da unidade de terapia intensiva de um hospital público da cidade de Feira de Santana, BA. Rev Soc Bras Clin Med. 2014;55(75).

23. Mirošević Skvrce N, Macolić Šarinić V, Mucalo I, Krnić $\mathrm{D}$, Božina N, Tomić S. Adverse drug reactions caused by drug-drug interactions reported to Croatian Agency for Medicinal Products and Medical Devices: a retrospective observational study. Croatian Medical Journal [Internet]. 2011;52(5):604-14. DOI: 10.3325/cmj.2011.52.604.

24. Brasil. Ministério da Saúde. Secretaria de Ciência, Tecnologia e Insumos Estratégicos. Departamento de Assistência Farmacêutica e Insumos Estratégicos. Formulário terapêutico nacional 2008: Rename 2006. Série B. Textos Básicos de Saúde. Brasília: Ministério da Saúde; 2008b. 
25. Olkkola KT, Ahonen J. Drug interactions. Current opinion in anaesthesiology [Internet]. 2001;14(4):411-6.

26. Neto VC, Garcia VP, Helena ET de S. Possible pharmacological interactions in hypertensive and/or diabetic elderly in family health units at Blumenau (SC). Brazilian Journal of Pharmaceutical Sciences. 2010;46(4):795-804. 10.1590/S1984-82502010000400022.

27. Bandeira VAC, de Oliveira KR. Potenciais interações entre medicamentos usados na síndrome metabólica. Scientia Medica. 2014;24(2):156-64.

28. Tan ECK, Stewart K, Elliott RA, George J. Pharmacist services provided in general practice clinics: A systematic review and meta-analysis. Vol. 10, Research in Social and Administrative Pharmacy. 2014. p. 608-22. DOI: 10.1016/j.sapharm.2013.08.006.

29. de Melo DO, de Castro LL. Pharmacist's contribution to the promotion of access and rational use of essential medicines in SUS. CIENCIA \& SAUDE COLETIVA. 2017;22(1):235-44. DOI: 10.1590/141381232017221.16202015 .

30. Brasil. Ministério da Saúde (MS). Departamento de Vigilância de Doenças e Agravos não Transmissíveis e Promoção de Saúde. Vigitel Brasil 2012: vigilância de fatores de risco e proteção para doenças crônicas por inquérito telefônico. Brasília: MS; 2013

31. Garcia LP, Sant’Anna AC, Magalhães LCG de, Freitas LRS de, Aurea AP. Gastos das famílias brasileiras com medicamentos segundo a renda familiar: análise da Pesquisa de Orçamentos Familiares de 2002-2003 e de 2008-2009. Cadernos de Saúde Pública [Internet]. 2013;29(8):160516.DOI: 10.1590/0102-311X00070912.

32. Correr CJ, Otuki, MF. A prática farmacêutica na farmácia comunitária. Porto Alegre: Artmed, 2013.

33. Obreli-Neto PR, Pereira LRL, Guidoni CM, De Baldoni AO, Marusic S, De Lyra DP, et al. Use of simulated patients to evaluate combined oral contraceptive dispensing practices of community pharmacists. PLoS ONE. 2013;8(12). DOI: 10.1371/journal.pone.0079875.

34. Brasil. Resolução da Diretoria Colegiada n. ${ }^{\circ} 44$ de 17 de agosto de 2009. Dispõe sobre Boas Práticas Farmacêuticas para o controle sanitário do funcionamento, da dispensação e da comercialização de produtos e da prestação de serviços farmacêuticos em farmácias e drogarias e dá outras providências. Diário Oficial da União, Brasília, DF, 18 ago. 2009. Seção 1, p.83.

35. Reis TM dos, Guidoni CM, Girotto E, Rascado RR, Mastroianni P de C, Cruciol JM, et al. Knowledge and conduct of pharmacists for dispensing of drugs in community pharmacies: A cross-sectional study. Brazilian Journal of Pharmaceutical Sciences. 2015;51(3):733-44. DOI: 10.1590/S1984-82502015000300025
36. International Pharmaceutical Federation. FIP. Standards for quality of pharmacy services. Good pharmacy practice. The Hague: FIP, 1993. Disponível em : https://www. fip.org/files/fip/Statements/latest/Dossier\%20004\%20total.PDF. Acesso em 24 de Outubro de 2017.

37. National Association of Pharmacy Regulatory Authorities. NAPRA. Model standards of practice for Canadian pharmacists. Ottawa: NAPRA, 2006.

38. Pharmaceutical Society of Australia. PSA. Standards for the provision of pharmacists only and pharmacy medicines in community pharmacies. Canberra: PSA, 1999.

39. Rabelo ML. Role of the pharmacist on the pharmacotherapeutic follow-up for cancer pain control ${ }^{*}$. 2013;14(1):5860. DOI:org/10.1590/S1806-00132013000100014.

40. Gregório J, Velez Lapão L. Uso de cenários estratégicos para planeamento de recursos humanos em saúde: o caso dos farmacêuticos comunitários em Portugal 2010-2020. Revista Portuguesa de Saude Publica. 2012;30(2):125-42. DOI10.1016/j.rpsp.2012.12.003.

41. Chisholm-Burns MA, Graff Zivin JS, Lee JK, Spivey CA, Slack M, Herrier RN, et al. Economic effects of pharmacists on health outcomes in the United States: A systematic review. American Journal of Health-System Pharmacy. 2010;67(19):1624-34. DOI: 10.2146/ajhp100077. 